\title{
Plasmonics-enabled metal-semiconductor-metal photodiodes for high-speed interconnects and polarization sensitive detectors
}

\author{
Evgeniy Panchenko ${ }^{a}$, Jasper J. Cadusch ${ }^{a}$, Timothy D. James ${ }^{\mathrm{a}}$, and Ann Roberts ${ }^{\mathrm{a}}$

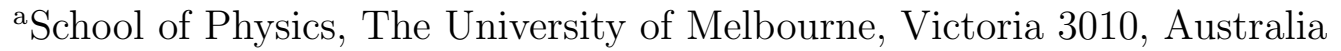

\begin{abstract}
Metal-semiconductor-metal (MSM) photodiodes are commonly used in ultrafast photoelectronic devices. Recently it was shown that localized surface plasmons can sufficiently enhance photodetector capabilities at both infrared and visible wavelengths. Such structures are of great interest since they can be used for fast, broadband detection. By utilizing the properties of plasmonic structures it is possible to design photodetectors that are sensitive to the polarization state of the incident wave. The direct electrical readout of the polarization state of an incident optical beam has many important applications, especially in telecommunications, bio-imaging and photonic computing. Furthermore, the fact that surface plasmon polaritons can circumvent the diffraction limit, opens up significant opportunities to use them to guide signals between logic gates in modern integrated circuits where small dimensions are highly desirable. Here we demonstrate two MSM photodetectors integrated with aluminum nanoantennas capable of distinguishing orthogonal states of either linearly or circularly polarized light with no additional filters. The localized plasmon resonances of the antennas lead to selective screening of the underlying silicon from light with a particular polarization state. The non-null response of the devices to each of the basis states expands the potential utility of the photodetectors while improving precision. We also demonstrate a design of waveguide-coupled MSM photodetector suitable for planar detection of surface plasmons.
\end{abstract}

Keywords: Photonics, CMOS, Schottky, Metal-semiconductor-metal, Metasurface, Plasmonics

\section{INTRODUCTION}

The utilization of beam polarization to code digital data, instead of, or in addition to, its intensity or phase can further increase the transmission rate and security of telecommunication systems. Nonetheless, despite these positive aspects, these systems are less robust to noise and require special techniques to reduce its influence.

Although optical systems are insensitive to electromagnetic noise they are still affected by several factors which decrease the signal-to-noise ratio. These include signal intensity fluctuations, the overlap of the spectral bandwidth of different channels and thermal wavelength drift. ${ }^{1}$ Additionally, a variety of nonlinear noise effects is introduced by dispersion and other nonlinear effects in optical fiber.

Differential detection approaches are often used to improve the robustness of a system to noise. ${ }^{2}$ In optical systems ${ }^{3}$ such interfaces can significantly reduce, or even eliminate, the influence of intensity and thermal fluctuations of a signal permitting a precise determination of beam polarization. In order to utilize a differential detection technique, one must compare the intensities of at least two orthogonal polarization states. Traditionally this would be done with the help of polarization optics such as a liquid crystal polarizer or a quarter wave plate. However, for modern electronic applications, minimizing device footprint is critical. Here we demonstrated a nanoscale alternative, capable of discriminating between optical signals with different polarizations which can, therefore, be used for differential optical detection ${ }^{4}$ (see Fig. 1).

Further author information: (Send correspondence to Evgeniy Panchenko)

Evgeniy Panchenko: E-mail: epanchenko@student.unimelb.edu.au 


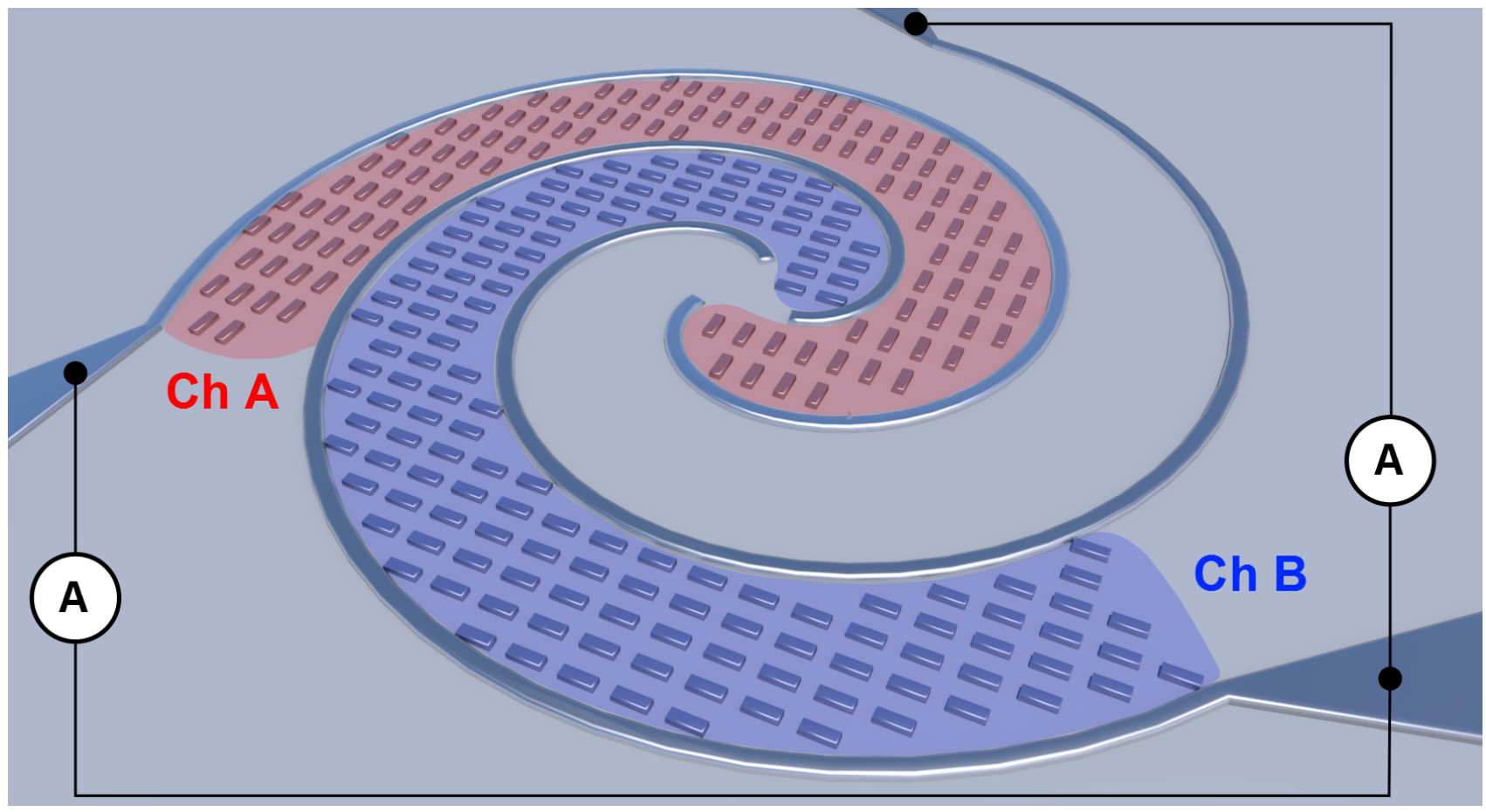

Figure 1. Schematic representation of a differential MSM photodetector with rectangular nanoantenna.

\section{DESIGN AND FABRICATION}

The device consists of three planar electrodes fabricated on Si and wrapped in a spiral configuration (see Fig. 3). Nanoantennas are fabricated between each pair of adjacent electrodes forming two photodiodes. Each contact forms a Schottky barrier with the Si substrate. The width of the contacts and the spacing between them were chosen to be $1.25 \mu \mathrm{m}$ and $1.5 \mu \mathrm{m}$ respectively. Here we consider simple nanorod antennas with dimensions $95 \times 45 \times 80 \mathrm{~nm}$. Other types of nanoantenna can alternatively be used to enable sensitivity to circularly ${ }^{5}$ or radially ${ }^{6}$ polarized light. The photocurrent in each photodetector is determined by direct absorption of photons in their active regions. The number of photons transmitted into the $\mathrm{Si}$ and, hence, absorbed is sensitive to the presence of nanoantennas on the surface and depends on their geometry. The nanoantennas in the active region of channel A (see Fig. 2a) are designed to respond to a particular polarization state leading to strong reflection, while the other set, in the active region of the channel B, responds to the orthogonal polarization state. As the orientation of the antennas between each pair of leads is different, there will be an imbalance between the photocurrents generated between each pair producing a polarization-dependent differential signal.

Photodetectors were fabricated on low doped [100] n-type silicon wafer with bulk resistance of $\rho=1-10$ $\Omega \cdot \mathrm{cm}$. Aluminum was used as a material for MSM contacts and pads as well as nanoantennas. Such choice of materials allows full compatibility with CMOS technology together with a low fabrication cost. The wafer was spincoated with $240 \mathrm{~nm}$ PMMA 950k resist and then exposed to create structures using a $100 \mathrm{kV}$ EBPG5000+ electron beam lithography system. The pattern was developed in 1:3 MIBK:IPA solution for 1 minute. The native oxide layer on the silicon substrate was stripped using $4 \%$ hydrofluoric acid and immediately loaded into a IntlVac NanoChrome II e-beam evaporator. The aluminum layer with a thickness of $85 \mathrm{~nm}$ was deposited at $0.7 \AA /$ s. After evaporation a lift-off step in pure acetone was performed. During the lift-off process the solution was heated to $60{ }^{\circ} \mathrm{C}$.

\section{EXPERIMENTAL RESULTS}

The normalized photocurrent from each photodetector at a wavelength of $625 \mathrm{~nm}$ as a function of HWP rotation angle is shown on Fig. 4. A full rotation of a HWP takes 8 min during which the laser source exhibited significant intensity fluctuations (random changes in the output signal). The photocurrent from the detectors can also vary 
a

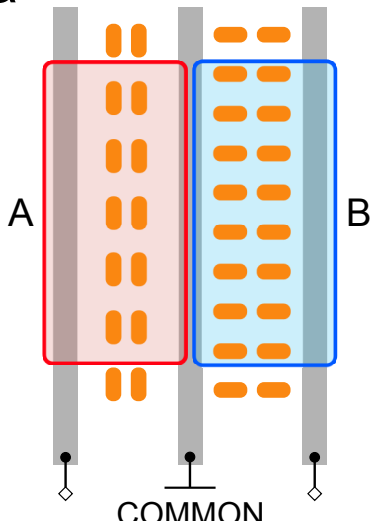

Ch A
COMMON

Ch B
B

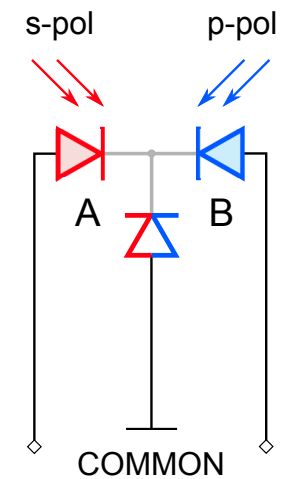

Ch A b

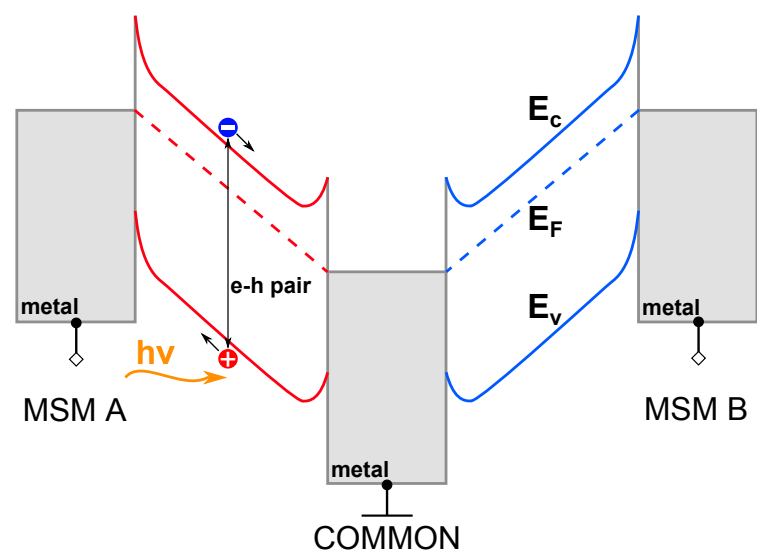

Figure 2. Schematic representation showing both channels of photodetector integrated with nanorods to sense linear polarization states (a). Active areas of channels (A and B) are formed between common and signal contacts. The equivalent electric circuit is represented by three Schottky diodes connected back-to-back. A band diagram under reverse bias and is shown on (b). $\mathrm{E}_{v}, \mathrm{E}_{c}$ and $\mathrm{E}_{F}$ are valence, conduction and Fermi energy levels respectively.

due to heating of the sample during measurements (a constant slope of the signal). These fluctuations are reflected in the raw signals (see Fig. 4 left) from both photodetectors.

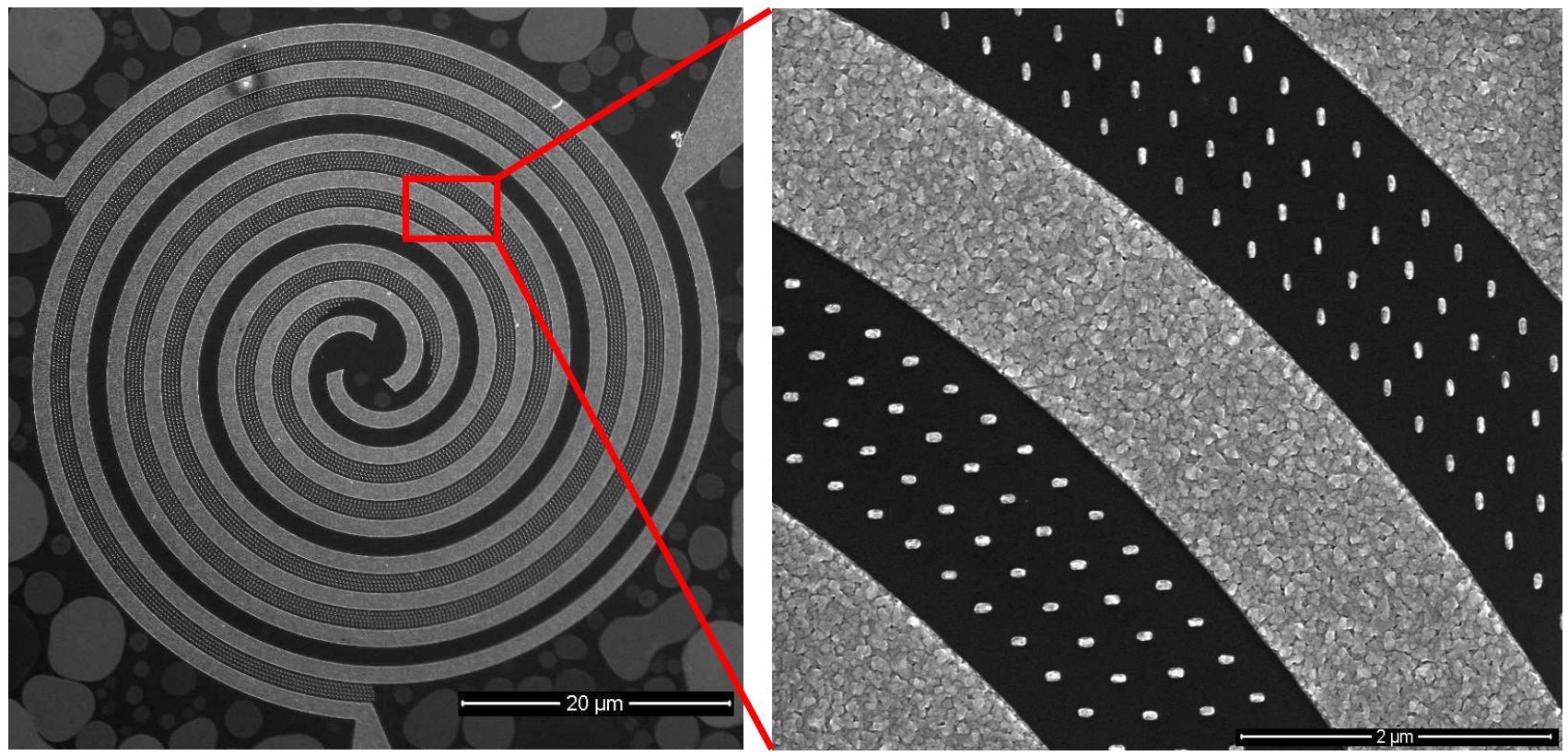

Figure 3. Scanning electron microscope image of the spiral photodetector. The inset shows two metasurfaces in the active regions of channels A and B designed to respond to orthogonal states of linear polarized light.

Although the average value of the signals from each photodetector individually varies, the differential photocurrent (see Fig. 4 right) has a relatively constant mean. Since the variation of power affects both photodetectors and remains in-phase, a simple subtraction of photocurrents eliminates the influence of this type of noise. An optical system utilizing differential spiral photodetectors, therefore, is relatively insensitive to fluctuations in signal intensity compared with conventional devices. This significantly increases the signal-to-noise ratio and, therefore, opens up the possibility of higher communication speeds. 

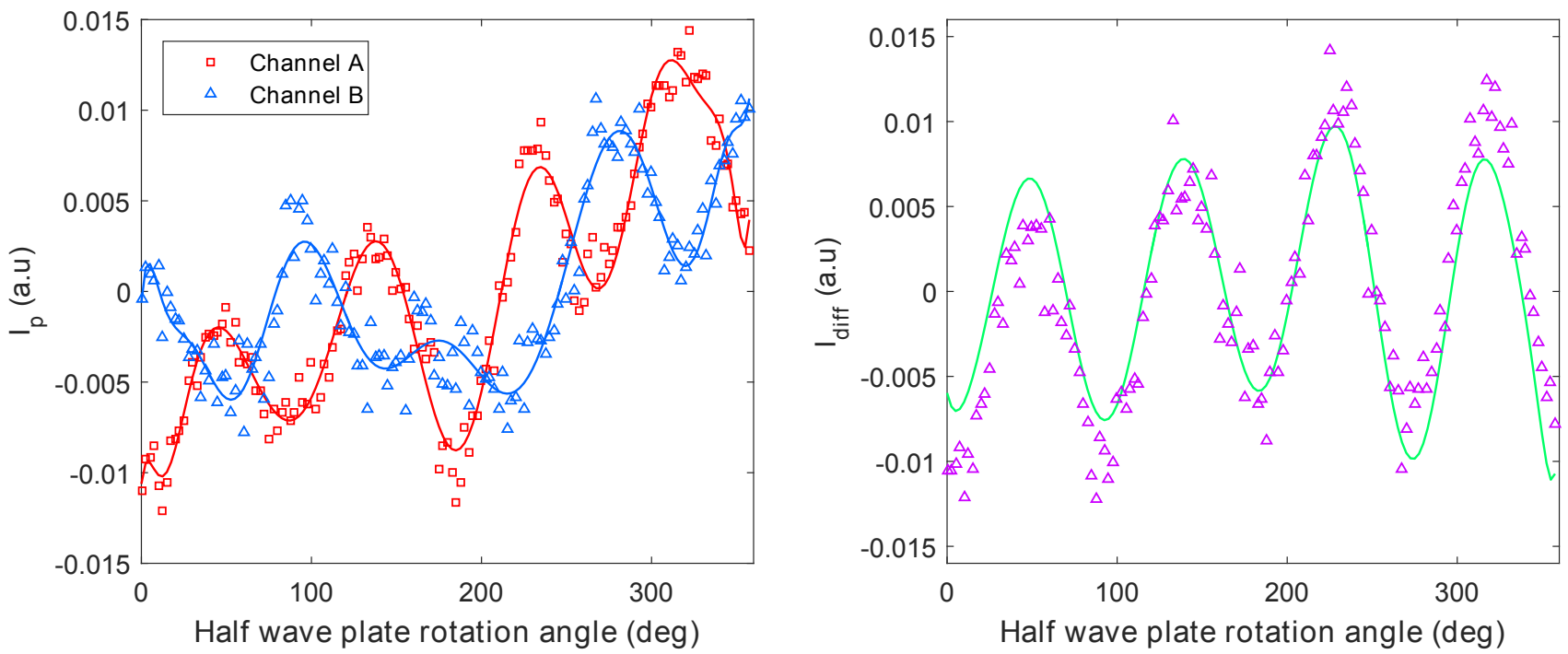

Figure 4. Normalized single-ended (left) and differential (right) photocurrents affected by a signal power fluctuation. As can be seen, utilization of differential detection technique decreases the influence of this type of noise.

\section{PLANAR SURFACE PLASMON DETECTION}

Surface plasmons are of great interest for digital electronics. A combination of nanoscale dimensions and strong field confinement, compared to the classic photonic elements, make utilization of the surface plasmons in integrated circuits more very attractive. ${ }^{7}$ The field of plasmonics, therefore, possess a greater potential to overcome the existing limitations and finally merge well developed microelectronics technology with promising photonic components.

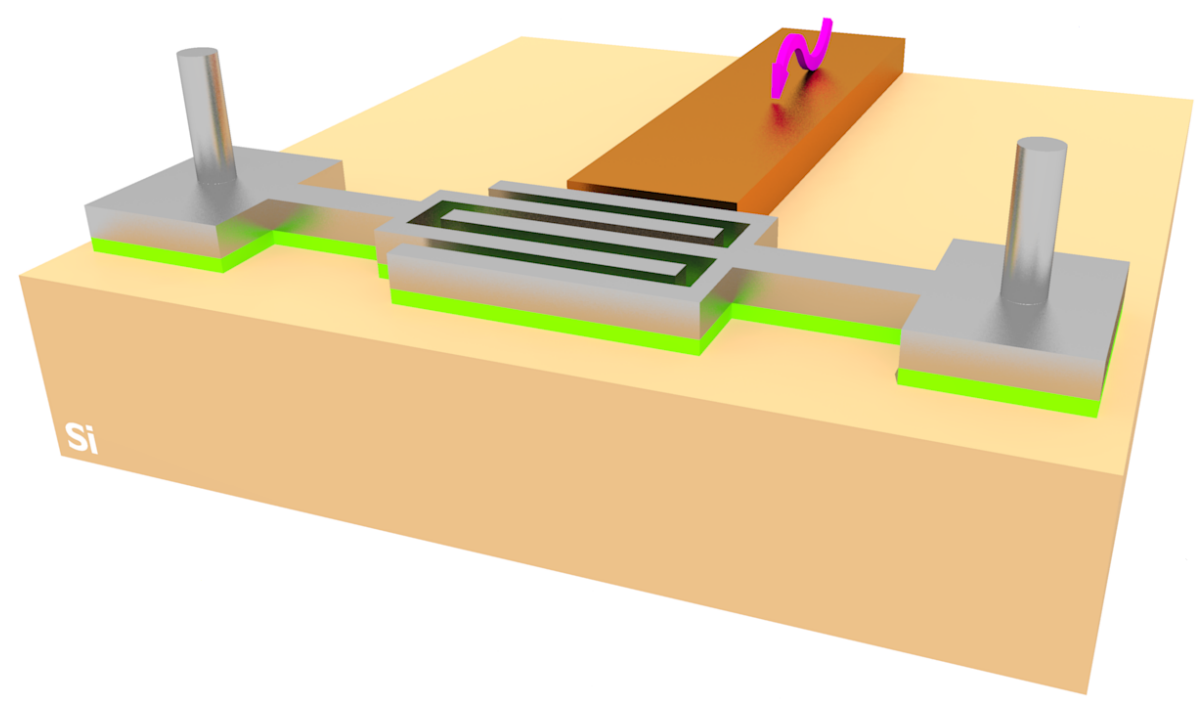

Figure 5. Schematic representation of a waveguide-coupled MSM photodetector.

In order to utilize surface plasmons as a signal carrier it is essential to provide a reliable detection mechanism.

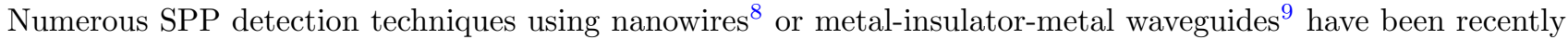
reported. Although these approaches showed a good capacity for detecting surface plasmons, their practical application is limited by complexity of design and fabrication. For smooth integration of plasmonic interconnects with detectors having a planar geometry and reproducible characteristics are essential. Here we propose a MSM photodiode coupled with a plasmonic waveguide (see Fig. 5) suitable for in-plane surface plasmon polariton 
detection.

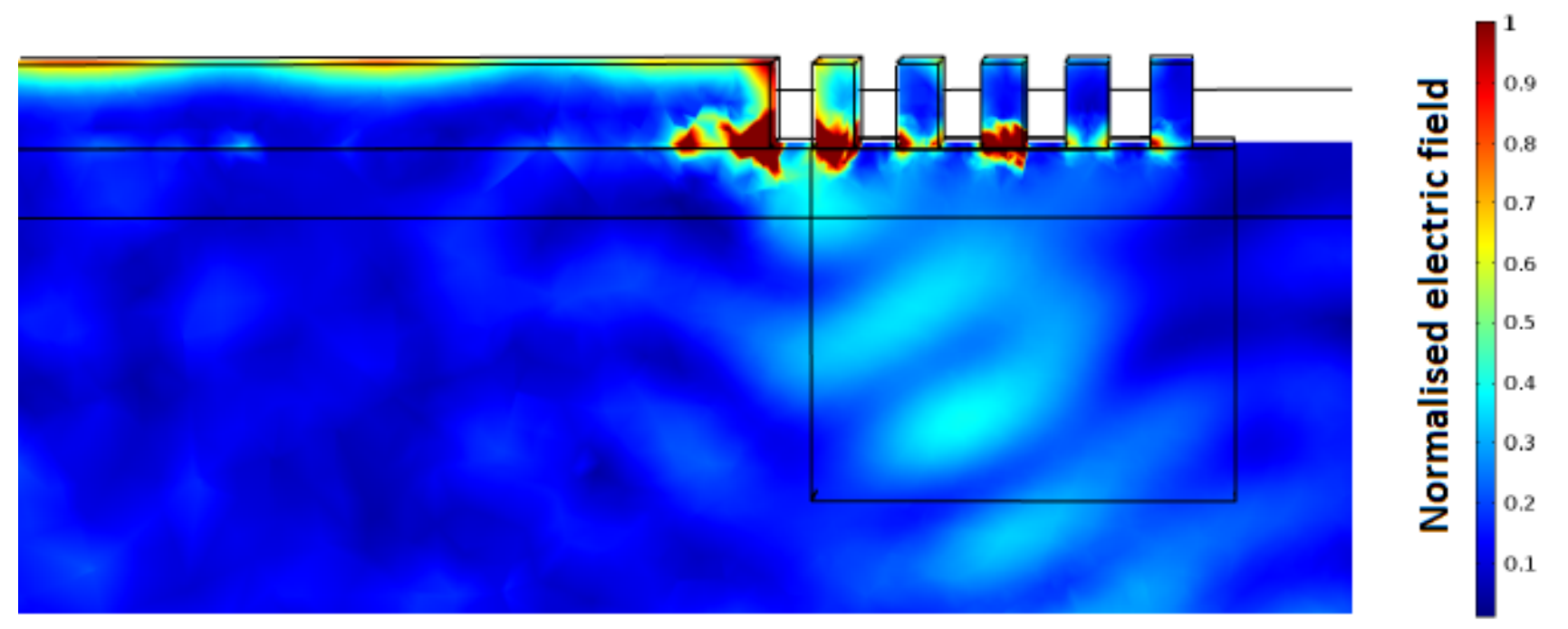

Figure 6. Normalized electric field map of the photodetector active region (under grating).

An MSM photodetector consists of two interdigitated metal electrodes (see Fig. 5) deposited on a low-doped semiconductor. Each of these electrodes forms a Schottky barrier with the substrate. The waveguide is used to deliver surface plasmons to the active zone of the MSM photodiode.

Several phenomena are simultaneously contributing to the photocurrent generated by this device. The fingers of the detector can couple surface plasmons back into the far-field (Fig. 6) allowing photons to penetrate into the substrate. If the photon energy is above the band gap of a semiconductor $\left(h \nu>E_{b g}\right)$ it will be absorbed resulting in electron-hole pair generation. The energy of generated electrons in this case is by definition (since the metal-semiconductor barrier is smaller than the bandgap of the semiconductor ${ }^{10}$ ) larger than the energy of the Schottky barrier and, therefore, can overcome this barrier. Furthermore, surface plasmons can nonradiatively decay in the metal fingers ${ }^{11}$ and be absorbed in them. This in turn, will lead to a hot-electron generation and injection through the Schottky barrier into the substrate. Therefore, such a device enables the detection of surface plasmons with energies above and below the band gap of the semiconductor.

To optimize the sensitivity of the device we performed an FEM simulation to determine the relationship between the irradiance delivered to the photodetector's active region, the separation between the fingers and their duty cycle. Increasing period of the fingers, while the duty cycle is kept fixed, increases the separation of the fingers, weakening the near-field coupling of each finger with its neighbors which, in turn, decreases the power flow into the substrate. The same tendency can be observed in the case of large duty cycles. This can be explained by the narrowing of the slits through which the surface plasmons outcouple into the substrate. Considering these results together with fabrication limitations we chose a grating with a duty cycle of 0.6 and with a period of $200 \mathrm{~nm}$. The photodetector consists of 13 fingers giving an active area of $12.5 \mu \mathrm{m}^{2}(5 \times 2.5 \mu \mathrm{m})$. Experimental realization and testing of this device is the subject of ongoing work. A device such as this could form one of the building blocks for high-speed optoelectronic integrated circuits.

\section{CONCLUSION}

In this paper we have demonstrated how surface plasmon phenomena can be used to enable new features in conventional photodetectors. It was shown that integration of plasmonic metasurfaces can enable polarization sensitivity in Schottky junction based photodetectors. A novel spiral photodetector design utilizing a differential detection approach which significantly improves signal-to-noise ration was also demonstrated. Additionally we proposed a design of a waveguide-coupled MSM photodetector which is capable of planar detection of surface plasmons. Such a design enhances the capacity of planar integration and is fully CMOS compatible. 


\section{ACKNOWLEDGMENTS}

This research was supported under the Australian Research Councils Discovery Projects funding scheme (project number DP110100221). This work was performed in part at the Melbourne Centre for Nanofabrication (MCN) in the Victorian Node of the Australian National Fabrication Facility (ANFF).

\section{REFERENCES}

[1] Cai, B., Johansson, L., Silva, C., Bennett, S., and Seeds, A. J., "Crosstalk, noise, and stability analysis of dwdm channels generated by injection locking techniques," Lightwave Technology, Journal of 21(12), 3029-3036 (2003).

[2] Narasimhan, A., Kasotiya, M., and Sridhar, R., "A low-swing differential signalling scheme for on-chip global interconnects," in [VLSI Design, 2005. 18th International Conference on], 634-639, IEEE (2005).

[3] Joshi, A., Wang, X., Mohr, D., Becker, D., and Wree, C., "Balanced photoreceivers for analog and digital fiber optic communications," in [Defense and Security], 39-50, International Society for Optics and Photonics (2005).

[4] Panchenko, E., Cadusch, J. J., James, T. D., and Roberts, A., "Plasmonic metasurface-enabled differential photodetectors for broadband optical polarization characterization," ACS Photonics (2016).

[5] Cadusch, J. J., James, T. D., Djalalian-Assl, A., Davis, T. J., and Roberts, A., "A chiral plasmonic metasurface circular polarization filter," Photonics Technology Letters, IEEE 26(23), 2357-2360 (2014).

[6] Chen, W., Abeysinghe, D. C., Nelson, R. L., and Zhan, Q., "Plasmonic lens made of multiple concentric metallic rings under radially polarized illumination," Nano letters 9(12), 4320-4325 (2009).

[7] Panchenko, E., James, T. D., and Roberts, A., "Modified stripe waveguide design for plasmonic input port structures," Journal of Nanophotonics 10(1), 016019-016019 (2016).

[8] Gramotnev, D. K. and Bozhevolnyi, S. I., "Plasmonics beyond the diffraction limit," Nature photonics 4(2), 83-91 (2010).

[9] Neutens, P., Van Dorpe, P., De Vlaminck, I., Lagae, L., and Borghs, G., "Electrical detection of confined gap plasmons in metal-insulator-metal waveguides," Nature Photonics 3(5), 283-286 (2009).

[10] Zimmermann, H., [Integrated silicon optoelectronics], Springer (2010).

[11] Sobhani, A., Knight, M. W., Wang, Y., Zheng, B., King, N. S., Brown, L. V., Fang, Z., Nordlander, P., and Halas, N. J., "Narrowband photodetection in the near-infrared with a plasmon-induced hot electron device," Nature communications 4, 1643 (2013). 


\section{University Library}

\section{- M I I N E R VA \\ A gateway to Melbourne's research publications}

Minerva Access is the Institutional Repository of The University of Melbourne

Author/s:

Panchenko, E;Cadusch, JJ;James, TD;Roberts, A

Title:

Plasmonics-enabled metal-semiconductor-metal photodiodes for high-speed interconnects and polarization sensitive detectors

Date:

2017-01-01

Citation:

Panchenko, E., Cadusch, J. J., James, T. D. \& Roberts, A. (2017). Plasmonics-enabled metal-semiconductor-metal photodiodes for high-speed interconnects and polarization sensitive detectors. GarciaBlanco, SM (Ed.) Conti, GN (Ed.) INTEGRATED OPTICS: DEVICES, MATERIALS, AND TECHNOLOGIES XXI, 10106, SPIE-INT SOC OPTICAL ENGINEERING. https://doi.org/10.1117/12.2250728.

Persistent Link:

http://hdl.handle.net/11343/243797 